\title{
Estimation of multiple objects at unknown locations with active contours
}

\author{
Margarida Silveira and Jorge S. Marques \\ IST - Instituto Superior Técnico, Technical University of Lisbon and ISR - Instituto \\ de Sistemas e Robótica, Portugal
}

\begin{abstract}
This paper presents an algorithm for the estimation of multiple regions with unknown shapes and positions using multiple active contour models (ACM's). The algorithm organizes edge points into strokes and computes the association between those strokes and the ACM's using the component wise EM algorithm (CEM) for MAP estimation. The algorithm is randomly initialized with a high number of ACM's and performs online model selection using importance sampling. Experimental results show the effectiveness of the proposed technique.
\end{abstract}

\section{Introduction}

Active contour models (ACM's) or snakes [1] have been extensively used to estimate object boundaries in images. However, their difficulties with initialization and outlier rejection are still unsolved problems. In addition, most of the research done on ACM's tries to estimate a single region using one elastic model (for e.g. see [2] [3]) and little research has addressed estimation of multiple elastic models. Some examples include [4] where multiple regions are estimated but the approach is restricted to regions that have some common characteristic or property and weighting parameters are defined heuristically. In [5] several ACM's are initialized in the centers of divergence of the gradient vector flow field. Some of the centers are discarded using heuristic rules and the method is unable to deal with regions inside other regions. In [6] a single contour can break automatically to represent the contours of multiple objects. In [7] multiple level set contours are also used but they evolve independently. The initial segmentation and number of ACM's is determined by fuzzy c-means clustering. In [8] gradient vector diffusion is used for the evolution and also the initialization of multiple contours. After the contours evolution region merging reduces the number of contours.

In this paper we present a method for the automatic segmentation of multiple regions which, in simultaneous with shape estimation, deals with the problem of sensitivity to the initialization and robustness to outliers. The algorithm builds on the work proposed in [9] in which multiple ACM's compete for the boundaries of multiple regions, using the EM algorithm for MAP estimation. The algorithm proposed in this paper includes three major contributions 1) it automatically selects the number of ACM's 2) it uses a different observation model which makes it less sensitive to initialization and more robust to outliers and 3) initialization of the ACM's is fully automatic. 
This paper is organized as follows: section 2 formulates the problem, section 3 describes the proposed algorithm for multiple active contours, section 4 presents experimental results and section 5 concludes the paper.

\section{Problem formulation}

Given an image with an unknown number of objects and assuming that is is possible to detect connected sets of edge points belonging to the objects boundaries, our aim is to connect segments belonging to individual object and to discard outlier segments associated with spurious edges. Let $y$ be the set of all edge points detected in an image and let us assume that $y$ is organized in connected components, called strokes, $y^{j}, j=1, \ldots, N$ where $y^{j}=\left\{y_{1}^{j}, \ldots, y_{n}^{j}\right\}$ is the set of edge points belonging to the $\mathrm{j}$-th stroke. We will assume for now that the number of ACM's, L is known and we add an extra model to account for outliers. We denote it the outlier model, $x^{\text {outlier }}$. Let $x^{k}$ be the the $\mathrm{k}$-th active contour model, $k=1, \ldots, L$ defined by a sequence of $2 \mathrm{D}$ points $x_{i}^{k}, i=1, \ldots, M^{k}$; the number of points for each snake is adjusted by insertion and deletion in order to keep the distance between two consecutive points constant and therefore different ACM's may have different number of points. $x^{k}$ can either be an open or closed contour. We will assume that the strokes detected in the image are independent:

$$
p(y \mid x)=\prod_{j} p\left(y^{j} \mid x\right)
$$

and that the distribution of each stroke is a mixture of $\mathrm{L}+1$ densities:

$$
p\left(y^{j} \mid x\right)=\sum_{k} \alpha_{k} p\left(y^{j} \mid x^{k}\right)+\alpha_{\text {outlier }} p\left(y^{j} \mid x^{\text {outlier }}\right)
$$

where the $\alpha_{k}$ 's are the mixing proportions verifying $\alpha_{k} \geq 0, \alpha_{\text {outlier }} \geq 0$ and $\sum_{k} \alpha_{k}+\alpha_{\text {outlier }}=1$.

Our aim is to estimate the ACM's and also their number L. We will iteratively estimate the ACM's using the MAP criterion and assuming L is fixed:

$$
x^{*}=\arg \max _{x} p(x \mid y)=\arg \max _{x}[\log p(y \mid x)+\log p(x)]
$$

Then a new value for $\mathrm{L}$ will be estimated by importance sampling. In the following we will specify each of the distributions involved in this problem.

\subsection{Observation and prior models}

We assume each stroke has i.i.d. edge points, each modelled by a mixture of $M^{k}$ Gaussian densities centered in the snake elements:

$$
p\left(y^{j} \mid x^{k}\right)=\prod_{n} p\left(y_{n}^{j} \mid x^{k}\right)=\prod_{n} \frac{1}{M^{k}} \sum_{i} N\left(y_{n}^{j}, x_{i}^{k}, \sigma^{2} I\right)
$$


where $N(y, \mu, R)$ denotes the normal density function with mean $\mu$ and covariance $R$. This model is closely related to the elastic net model [10] and associates every edge point with a given snake element. For the case of the outlier model, the contribution of each feature to the potential is a constant, but a different constant is used for each stroke, $V^{j}$.

$$
p\left(y^{j} \mid x^{\text {outlier }}\right)=\prod_{n} p\left(y_{n}^{j} \mid x^{\text {outlier }}\right)=\prod_{n} N^{j} V^{j}=\left(V^{j}\right)^{N^{j}}
$$

If $V^{j}$ is set inversely proportional to the size of the corresponding stroke, $N^{j}$, then the smaller strokes will also tend to be classified as outliers, and the ACM's will be able to bridge the small outlier strokes. Therefore we used $V^{j}=$ $\exp \left(-K N^{j}\right)$ where $\mathrm{K}$ is a positive constant.

We adopt the prior model proposed in [9] which is the following:

$$
\log p(x)=\sum_{k}\left(E_{\mathrm{int}}\left(x^{k}\right)+\sum_{l \neq k} E_{\mathrm{inter}}\left(x^{k}, x^{l}\right)\right)
$$

where $E_{\mathrm{i}}\left(x^{k}\right)$ is a regularization energy that expresses the assumption that each contour is smooth and $E_{\text {inter }}\left(x^{k}, x^{l}\right)$ is another regularization energy that expresses the interaction between different active contours.

\section{Unsupervised multiple active contours estimation}

The algorithm proposed in [9] described the estimation of multiple models in which multiple ACM's compete for the boundaries of the multiple regions. The algorithm solves the association between strokes and multiple models problem and also the outlier rejection. However it does not solve the initialization problem and the estimation of the number of models. To deal with these difficulties we initiate the algorithm with an arbitrary large number of snakes, L. The initialization of these L ACM's is fully automatic; circular ACM's are randomly distributed throughout the image, inside the strokes bounding boxes. The size of the circles is defined by the average size of the bounding boxes or may be user defined. Then the algorithm iteratively performs the following two steps.

1. Update

The ACM's are sequentially updated with the Component wise EM algorithm which is summarized in the sub-section 3.1. Convergence is achieved when all the points move less than a threshold.

2. Sampling

The algorithm relocates the ACM's by performing Importance Sampling using the mixing proportions $\alpha_{k}$ as the importance function. The set of ACM's $x^{k}, k=1, \ldots L$ is sampled in order to obtain a new set of L ACM's with the highest values of $\alpha_{k}$. Obviously some ACM's will be sampled several times and other will not be sampled at all. The ACM's that are not sampled 
are eliminated and the ones that were sampled several times will give rise to new ACM's that are equal. However the CEM algorithm will insure they will converge to different locations since they are updated one at a time.

The sampling step does not change the number of ACM's. Therefore, in order to reduce the number of ACM's, we add a model elimination step every $\mathrm{P}$ iterations. In this step we eliminate multiple copies of the models which were sampled several times and keep only one realization of such ACM. The number of different ACM's is the estimated number of models.

\subsection{Component Wise EM algorithm}

In the EM algorithm it is assumed that $\mathrm{y}$ is incomplete data and that the complete data includes binary labels $z_{j}, j=1, \ldots, N$ with $z_{j}=\left\{z_{j}^{1}, \ldots, z_{j}^{L+1}\right\}$, that indicate which model generated the stroke; $z_{j}^{k}=1$ means that stroke $y^{j}$ was generated by model $x^{k}$. The complete log likelihood is given by:

$$
\log p(y, z \mid x)=\sum_{j} \sum_{k} z_{j}^{k} \log p\left(y^{j} \mid x^{k}\right)
$$

Instead of maximizing (3), the EM algorithm alternates between two steps. In the E-step it finds the conditional expectation of the complete log likelihood with respect to the unknown $x$ given the observed data $y$ and the current estimate, $\hat{x}$.

$$
\begin{gathered}
Q(x, \widehat{x})=E[\log p(y, z \mid x) \mid y, \widehat{x}] \\
=E\left[\sum_{j} \sum_{k} z_{j}^{k} \log \left[\alpha_{k} p\left(y^{j} \mid x^{k}\right)\right]\right]=\sum_{j} \sum_{k} w_{k}^{j} \log \left[\alpha_{k} p\left(y^{j} \mid x^{k}\right)\right]
\end{gathered}
$$

where $w_{k}^{j}$ is a set of weights summing to one assigned to each stroke. Each weight $w_{k}^{j}$ represents the soft assignment of stroke $y^{j}$ to the active contour $x^{k}$. The weights are given by:

$$
w_{k}^{j}=p\left(z_{j}^{k}=1 \mid y^{j}, \widehat{x}\right)=\frac{\alpha_{k} p\left(y^{j} \mid x^{k}\right)}{\sum_{m} \alpha_{m} p\left(y^{j} \mid x^{m}\right)}
$$

In the M-step the estimation of the active contour is obtained by the maximization of:

$$
U(x, \widehat{x})=Q(x, \widehat{x})+\log p(x)
$$

The CEM algorithm sequentially performs one E step and one $\mathrm{M}$ step for each of the ACM's and iterates until convergence [11]. In our implementation the order of this estimation is predefined.

The E and M steps will be detailed in the following subsections. 
The E-Step In the E-step the weights are calculated. Substituting (4) into (10) we obtain the following expression:

$$
\mathrm{w}_{\mathrm{k}}^{\mathrm{j}}=\frac{\alpha_{k} \prod_{n} \frac{1}{M^{k}} \sum_{i} \mathrm{~N}\left(x_{i}^{k}, \sigma^{2} I\right)}{\sum_{m} \alpha_{m} \prod_{n} \frac{1}{M^{m}} \sum_{i} \mathrm{~N}\left(x_{i}^{m}, \sigma^{2} I\right)+\alpha^{\text {outlier }}\left(V^{j}\right)^{N^{j}}}
$$

The mixing proportions are updated by:

$$
\alpha_{k}=\frac{1}{N^{j}} \sum_{j} w_{k}^{j}
$$

The M-Step In the M-step the estimation of the active contour is obtained by the minimization of (11) performed by the gradient algorithm:

$$
x_{t+1}^{k}=x_{t}^{k}-\gamma \nabla_{x}(Q(x, \widehat{x}))
$$

where $\nabla_{x}$ represents the gradient. This equation can be rewritten as follows:

$$
x_{t+1}^{k}=x_{t}^{k}-\gamma_{\text {int }} f_{\text {int }}-\gamma_{\text {ext }} f_{\text {ext }}-\gamma_{\text {inter }} f_{\text {inter }}
$$

where $f_{\text {ext }}\left(x_{i}^{k}\right), f_{\text {int }}\left(x_{i}^{k}\right)$ and $f_{\text {inter }}\left(x_{i}^{k}\right)$ are external, internal and interaction forces. External and internal forces are given by expressions (16) and (17):

$$
\begin{gathered}
f_{\text {ext }}\left(\mathrm{x}_{\mathrm{i}}^{\mathrm{k}}\right)=-\frac{1}{\sigma^{2}} \sum_{j} w_{j}^{k} \sum_{n}\left(y_{n}^{j}-x_{i}^{k}\right) \phi_{\sigma}\left(\left|y_{n}^{j}-x_{i}^{k}\right|^{2}\right) \\
f_{\text {int }}\left(x_{i}^{k}\right)=-2\left(\frac{l_{i-1}-l_{0}}{l_{i-1}}\left(x_{i}^{k}-x_{i-1}^{k}\right)+\frac{l_{i+1}-l_{0}}{l_{i+1}}\left(x_{i}^{k}-x_{i+1}^{k}\right)\right)
\end{gathered}
$$

where $l_{i-1}=\left\|x_{i}^{k}-x_{i-1}^{k}\right\|$ and $l_{i+1}=\left\|x_{i}^{k}-x_{i+1}^{k}\right\|$. The expression of the interaction force depends on the application. For instance, if we expect each model to attract the other models, we can use $\varphi(d)=-\exp \left(-d / 2 \sigma_{\text {inter }}^{2}\right)$ leading to:

$$
f_{\text {inter }}\left(\mathrm{x}_{\mathrm{i}}^{\mathrm{k}}\right)=\frac{1}{\sigma_{\text {inter }}^{2}} \sum_{l \neq k} \sum_{m}\left(x_{i}^{k}-x_{m}^{l}\right) \varphi\left(\left|x_{m}^{l}-x_{i}^{k}\right|^{2}\right)
$$

\section{Experimental Results}

This section presents examples to illustrate the performance of the proposed method. The examples were performed in the following conditions. Edges were obtained with the Canny edge detector and strokes were obtained with a connected components labelling algorithm. The external forces acting on each model unit were multiplied by independent gains to limit the maximum displacement of the model units in each iteration. All the experiments used $\Delta_{\max }=2$ and the gain factors $\gamma_{\text {int }}$ and $\gamma_{\text {inter }}$ were chosen manually. 

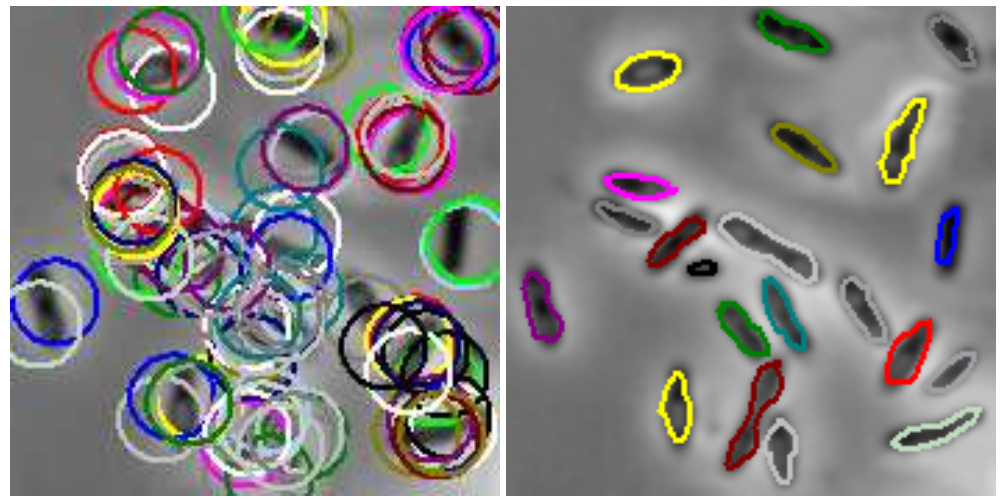

Fig. 1. Bacteria Example; 100 initial contours (left) and 21 final estimated contours (right).

The first example illustrates the performance of the algorithm in the presence of multiple objects (bacteria). Fig. 1 shows the initial contours on the left and the final contours on the right. The algorithm was initialized with 100 ACM's that were overlapping and the final result was able to separate 21 different objects. All the objects were correctly associated with a different ACM.

The second example shows the performance of the proposed algorithm applied to the segmentation of pedestrians in a video sequence. In this example 10 ACM's were used to segment the image obtained from Fig. 2 a) after background subtraction. The background estimation was based on modelling the intensity of each pixel with a single gaussian. Fig. 2 shows the initial contours on the left and the final contours on the right. The algorithm successfully estimated the correct number of pedestrians, producing 4 ACM's.
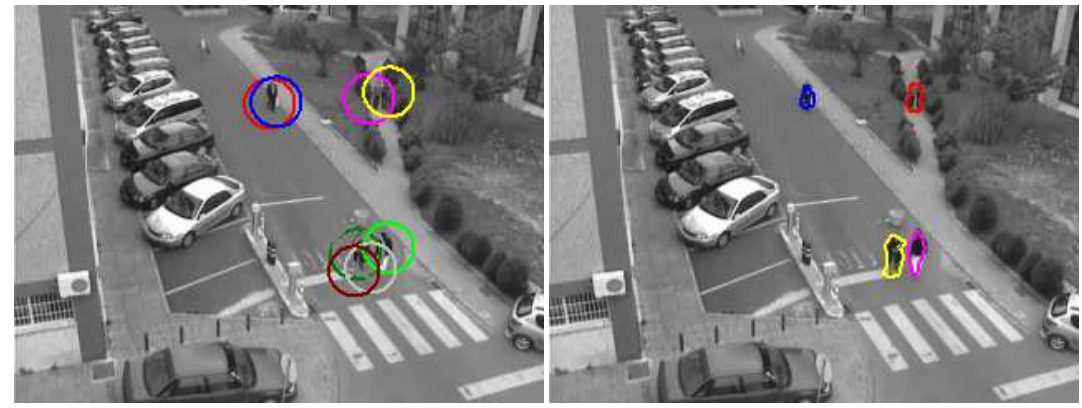

Fig. 2. Pedestrians; 10 initial contours (left) and 4 final estimated contours (right).

The third example shows the performance of the proposed algorithm with nested regions. In this example 50 ACM's were used to segment inhibition halos 
of antibacterial activity in microbiologic plate assays. Fig. 3 shows the initial contours on the left and the final contours on the right. The algorithm successfully estimated the correct number of objects, producing 12 ACM's and detecting no outliers.

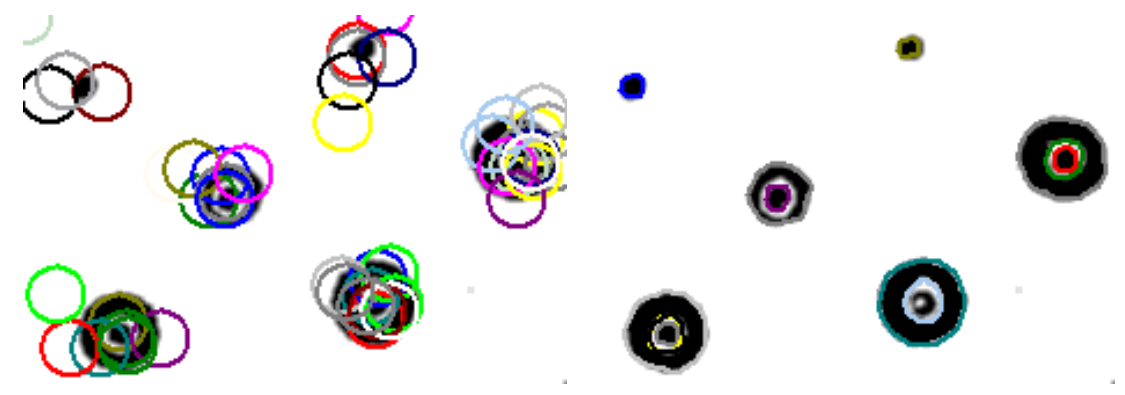

Fig. 3. Microbiologic plate assay; 50 initial contours (left) and 12 final estimated contours (right).

The final example illustrates the application of the algorithm to a blood cell image using the outlier model to discard the smaller objects. The algorithm was initialized with 120 ACM's and in the final segmentation 27 contours remain. Fig. 4 shows the initial contours on the left, the final contours in the middle and the strokes classified as outliers on the right. In this example a couple of the final ACM's represent more than one object because they were overlapping in the image and originated only one stroke. The outliers that were detected correspond to the smaller strokes present in the image.

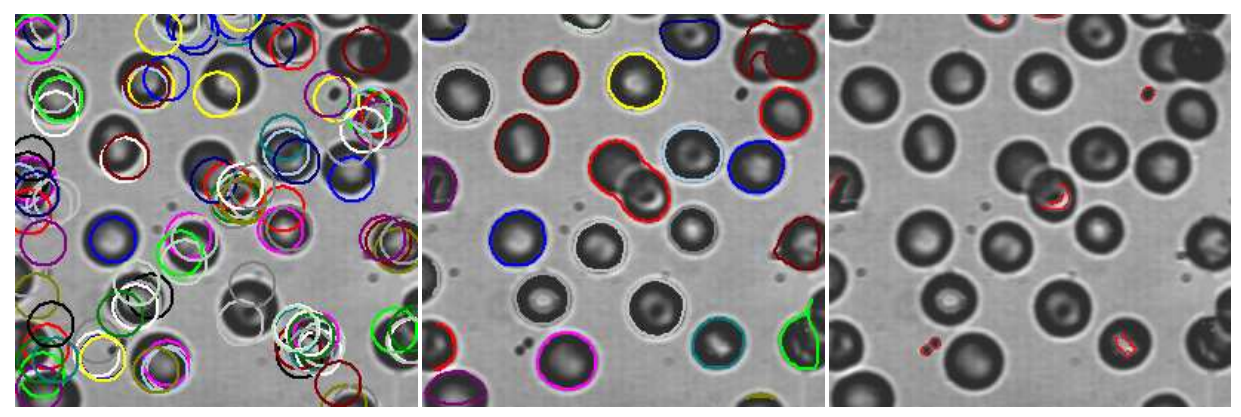

Fig. 4. Cell example; 120 initial contours (left), 27 contour estimates (middle) and outlier strokes (right). 


\section{Conclusions}

This paper presents an algorithm for the extraction of multiple regions using multiple active contour models (ACM's). Initialization is automatic, the algorithm estimates the number of models and also accounts for outlier features detected in the image. It is shown that the proposed algorithm is able to robustly estimate all the deformable contours and to compute the association probability between strokes and multiple models.

Acknowledgments This work was supported by Fundação para a Ciência e a Tecnologia (ISR/IST plurianual funding) through the POS_Conhecimento Program that includes FEDER funds.

\section{References}

[1] Kass, M.; Witkin, A. and Terzopoulos, D., Snakes: Active contour models, International Journal of Computer Vision. 1(4): 321 331, 1987.

[2] Cohen,L., On active contour models and balloons, CVGIP: Image Understanding, 53(2): 212-218, 1991.

[3] Xu, C. and Prince, J. L., Snakes, shapes, and gradient vector flow, IEEE Trans. on Image Processing, 7(3):359-369, 1998.

[4] Srinark,T. and Kambhamettu, C., A framework for multiple snakes, Proceedings of the 2001 IEEE Computer Society Conference on Computer Vision and Pattern Recognition, II: 202-209, 2001.

[5] Xingfei, G. and Jie, T.; An automatic active contour model for multiple objects, Proceedings of the 16th International Conference on Pattern Recognition, 2: 881884, 2002.

[6] Choi, W.; Kin-Man, L. Siu,W., An adaptive active contour model for highly irregular boundaries,Pattern Recognition 34: 323-331, 2001.

[7] Schupp, s.; Elmoataz, A.; Fadili, J. ; Herlin, P. , Bloyet, D., Image Segmentation via Multiple Active Contour Models and Fuzzy Clustering with Biomedical Applications, International Conference on Pattern Recognition (ICPR'00), 1: 622-625, 2000 .

[8] Yu, Z. and Bajaj, c., Image Segmentation Using Gradient Vector Diffusion and Region Merging,International Conference on Pattern Recognition (ICPR'02), 2: 941944, 2002.

[9] Silveira, M. and Marques, J., Multiple Active Contour Models based on the EM algorithm, IEEE International Conference on Image Processing, ICIP 2005, Genova, Italy, September 2005.

[10] Durbin, R. and Willshaw, D.; An analogue approach to the travelling salesman problem using an elastic net method, Nature. 326 :689-91, 1987

[11] Celeux, G., Chréetien, Forbes, F. and Mkhadri, A. A component-wise EM algorithm for mixtures, Journal of Computational and Graphical Statistics, 10: 699-712, 2001. 